\title{
Utilisation de l'intelligence artificielle en pharmacie : une revue narrative
}

\author{
par Laura Gosselin, Maxime Thibault, Denis Lebel et Jean-François Bussières
}

J. Can. Pharm. Hosp. 2021;74(2):135-43

\section{RÉSUMÉ}

Contexte : L'intelligence artificielle (IA) est une avancée technologique qui consiste à amener une machine à imiter une forme d'intelligence.

Objectifs : L'objectif principal est d'effectuer une revue narrative des études évaluant la faisabilité et l'impact de I'IA en pharmacie. L'objectif secondaire est de développer une carte heuristique entourant I'IA en santé.

Sources des données : Nous avons consulté quatre bases de données, soit PubMed, Medline, Embase et CINAHL.

Sélection des études et extraction des données : Quatre stratégies de recherche ont été élaborées. Sélection des articles sur la base du titre, de l'abrégé puis du texte par une assistante de recherche, suivie d'une révision par un pharmacien de l'équipe. Les articles pris en compte doivent décrire ou évaluer la faisabilité ou l'impact de l'IA en pharmacie.

Synthèse des données : À partir de la revue documentaire, 362 articles ont été sélectionnés au départ, 18 d'entre eux ont été retenus selon les critères d'inclusion. De façon générale, on note que les études ont été surtout menées aux États-Unis (72\%, 13/18). Les études portent, par ordre d'importance décroissant, sur la prédiction de la réponse aux traitements et la prédiction d'effets indésirables $(33 \%, 6 / 18)$, la priorisation des patients (28 \%, 5/18), l'adhésion thérapeutique $(22 \%, 4 / 18)$, la validation d'ordonnances et la prescription électronique $(17 \%, 3 / 18)$ et d'autres thèmes (p. ex. diagnostic, coûts, assurance, vérification de volumes de seringue).

Conclusions : Cette revue narrative met en évidence 18 études évaluant la faisabilité et l'impact de I'IA en pharmacie. Ces études ont utilisé différentes approches méthodologiques dans divers domaines d'application, en officine comme en établissement de santé. Il est encore trop tôt pour prédire les retombées de I'IA en pharmacie, mais ces études soulignent l'importance de s'y intéresser.

Mots-clés : pharmacie, intelligence artificielle, revue narrative

\section{INTRODUCTION}

Lintelligence artificielle (IA) est une avancée technologique qui consiste à amener une machine à imiter une forme d'intelligence. Le Grand Dictionnaire terminologique précise qu'il sagit d'un " domaine d'étude ayant pour objet la reproduction artificielle des facultés cognitives de l'intelligence humaine dans le but de créer des systèmes ou des machines capables d'exécuter des fonctions relevant normalement de celle-ci. L'IA touche à de nombreux domaines, comme les sciences cognitives et les mathématiques, et à diverses

\begin{abstract}
Background: Artificial intelligence (Al) can be described as an advanced technology in which machines display a certain form of intelligence.

Objectives: The primary objective was to perform a narrative review of studies evaluating the feasibility and impact of Al in pharmacy. The secondary objective was to create a mind map of $\mathrm{Al}$ in health care.

Data Sources: Four databases were consulted: PubMed, Medline, Embase, and CINAHL.

Study Selection and Data Extraction: Four search strategies were developed. Initial selection of articles was based on their titles and abstracts; the full texts were then evaluated by a research assistant, with review by a pharmacist. Articles were included if they described or evaluated the feasibility or impact of Al in pharmacy.
\end{abstract}

Data Synthesis: A total of 362 articles were identified by the literature review, of which 18 met the inclusion criteria. The studies were mainly conducted in the United States $(72 \%, 13 / 18)$. The article topics were, in decreasing order, prediction of response to treatments and adverse effects $(33 \%, 6 / 18)$, patient prioritization $(28 \%, 5 / 18)$, treatment adherence $(22 \%, 4 / 18)$, validation of prescriptions and electronic prescription (17\%, 3/18), and other themes (e.g., diagnosis, costs, insurance, and verification of syringe volume).

Conclusions: This narrative review highlighted 18 studies evaluating the feasibility and impact of $\mathrm{Al}$ in pharmacy. The studies used various methodologies in different settings, both retail pharmacies and hospital pharmacies. It is still too soon to predict the implications of Al for pharmacy, but these studies emphasize the importance of attention in this area.

Keywords: pharmacy, artificial intelligence, literature review

applications, notamment en reconnaissance des formes, en résolution de problèmes, en robotique, dans les jeux vidéo ainsi que dans les systèmes experts ${ }^{1}$. L'Office québécois de la langue française propose un lexique de 85 termes associés à l'IA ${ }^{2}$.

Dans le domaine de la santé, l'IA a été utilisée en imagerie médicale afin d'assister la reconnaissance visuelle d'images recueillies lors d'examens radiologiques; ${ }^{3}$ elle a également été utilisée pour l'optimisation de choix de traitement en oncologie $e^{4}$. 
Compte tenu de l'informatisation de toutes les ordonnances des patients dans les dossiers pharmacologiques depuis quelques décennies, tant en officine qu'en établissement de santé, ces bases de données représentent une réelle occasion de développer des modèles d'apprentissage automatique afin de prédire des éléments utiles à la pratique pharmaceutique. Les données provenant des dossiers médicaux électroniques sont évidemment à l'avant-plan des sources utiles au développement de l'IA et peuvent être combinées aux données tirées de la pratique de la pharmacie.

Flynn souligne qu'il existe différentes possibilités mais également de la confusion entourant les concepts et les termes utilisés ainsi que plusieurs craintes liées à l'utilisation de l'IA dans la pratique pharmaceutique ${ }^{5}$.

Puisqu'il s'agit d'un sujet d'actualité en émergence et que l'IA représente une opportunité en pharmacie, nous nous sommes intéressés à l'utilisation de l'IA dans la pratique pharmaceutique.

L'objectif principal était d’effectuer une revue narrative des études évaluant l'utilisation de l'IA en pratique pharmaceutique.

\section{MÉTHODES}

\section{Sources des données et sélection des études}

Dans un premier temps, nous avons recensé les concepts, les termes et les applications de l'IA en pharmacie à partir d'articles d'intérêt général, d'une séance de remue-méninges et de travaux préliminaires. Sur la base des termes obtenus, nous avons développé par itération une carte heuristique des termes applicables à l'IA en pharmacie. Ces travaux préliminaires et cette carte heuristique ont contribué à clarifier le vocabulaire pertinent pour notre stratégie de recherche.

Dans un deuxième temps, nous avons mené une recherche sur les études évaluant l'utilisation de l'IA en pratique pharmaceutique. Nous avons consulté quatre bases de données, soit PubMed, Medline, Embase et CINAHL.

Dans PubMed, nous avons utilisé la stratégie de recherche suivante : ((((Pharmacy Service, Hospital) $\mathrm{OR}$ " Pharmacy " $[\mathrm{MeSH}])$ OR " Community Pharmacy Services » $[\mathrm{MeSH}])$ AND « Artificial Intelligence » $[\mathrm{MeSH}])$.

Dans Embase, nous avons utilisé la stratégie de recherche suivante : (artificial intelligence/ or artificial neural network/ or network learning/ or learning algorithm/ or supervised machine learning/ or unsupervised machine learning/ or support vector machine/ or exp Machine learning/ or (AI or IA or neural network * or ((deep or machine) and learning)).ti,ab,kw. AND pharmacy/ or hospital pharmacy/ or clinical pharmacy/).

Dans CINAHL, nous avons utilisé la stratégie de recherche suivante : ( $\mathrm{MH}$ (artificial intelligence OR deep learning OR machine learning) OR TI(AI OR IA or neural network $^{\star}$ or ((deep or machine) and learning)) OR SO(AI or IA or neural network ${ }^{*}$ or ((deep machine) and learning)) AND TI(pharmacy OR hospital pharmacy service* OR clinical pharmacy OR community pharmacy service ${ }^{\star}$ OR $\mathrm{SO}$ (pharmacy OR hospital pharmacy service* OR clinical pharmacy OR community pharmacy service $\left.{ }^{\star}\right)$ ).

Chaque stratégie de recherche a nécessité que nous sélectionnions les articles sur la base du titre, puis de l'abrégé puis du texte complet. Les abrégés de conférences pertinents ont aussi été retenus. La sélection a été effectuée par une assistante de recherche et par un pharmacien de léquipe. Les divergences ont été résolues par consensus. Les articles retenus devaient décrire ou évaluer l'utilisation de l'IA dans un domaine applicable à la pratique de la pharmacie. Compte tenu de l'émergence de cette discipline, nous nous sommes intéressés à deux dimensions plus spécifiques, soit la faisabilité de recourir à l'IA en pharmacie et les mesures d'impact de son utilisation. Ont été exclus les articles sans rapport avec l'IA, qui n'avaient pas de données originales, qui ne parlaient pas d'utilisation applicable en pratique pharmaceutique en milieu communautaire ou hospitalier.

\section{Extraction des données}

À partir des articles retenus, nous avons constitué un tableau synthèse de lecture comportant le premier auteur, l'année de publication, le pays, le type détude, les objectifs, la description de l'utilisation de l'IA en pharmacie, la taille de léchantillon $(n)$, les principaux résultats, les limites et la pertinence de leur utilisation dans la pratique pharmaceutique. Le tableau synthèse nous a permis de déterminer les réussites et les difficultés liées à l'utilisation de l'IA en pharmacie.

Aucune analyse statistique n’a été menée.

\section{SYNTHÈSE DES DONNÉES}

La figure 1 illustre la carte heuristique des termes applicables à l'IA en santé. Ainsi, l'IA repose sur l'utilisation d'un ensemble de données quoon peut exploiter à partir de différentes méthodes, dont l'apprentissage automatique dans une variété de champs d'application. L'utilisation de ces données comporte des enjeux éthiques. Cette carte heuristique aide à la mise en contexte des études retenues dans notre revue narrative.

À partir de la revue documentaire, nous avons sélectionné 362 articles au départ et retenu 18 selon les critères d'inclusion. Après l'exclusion des articles sur la base du titre et du résumé, il en restait 33, dont 15 ont été exclus. Trois étaient des doublons et 12 ont été exclus sur la base du texte complet, car ils ne comportaient pas de données originales, n'avaient pas de rapport avec l'IA, ne parlaient pas de pharmacie ou ne s'appliquaient pas à la pharmacie communautaire ou hospitalière. Enfin, l'un des articles compris dans cette revue narrative est une version améliorée d'un article exclu de notre sélection et publié par le même auteur 6 .

La figure 2 présente la cartographie de sélection des articles inclus et exclus.

De façon générale, on note que les études ont été surtout menées aux États-Unis $(72 \%, 13 / 18)$. 
Elles portent, par ordre d'importance décroissant, sur la prédiction de la réponse aux traitements et la prédiction d'effets indésirables $(33 \%, 6 / 18)$, la priorisation des patients $(28 \%$, $5 / 18)$, l'adhésion thérapeutique $(22 \%, 4 / 18)$, la validation d'ordonnances $(17 \%, 3 / 18)$ et d'autres thèmes (p. ex. diagnostic, coûts, assurance, vérification de volumes de seringue).

En ce qui concerne la réponse aux traitements et la prédiction d'effets indésirables, les études ont en général pour but d'aider à prédire la capacité du patient à répondre ou non à un traitement, le risque de développer un effet indésirable ou les paramètres pharmacocinétiques.

En ce qui a trait à la priorisation des patients, les études se concentrent sur la sélection de patients dans le but d'offrir une intervention à ceux qui ont le plus de chances d’en bénéficier. Ces études sont nombreuses à offrir des interventions ciblant l'adhésion thérapeutique, mais d'autres

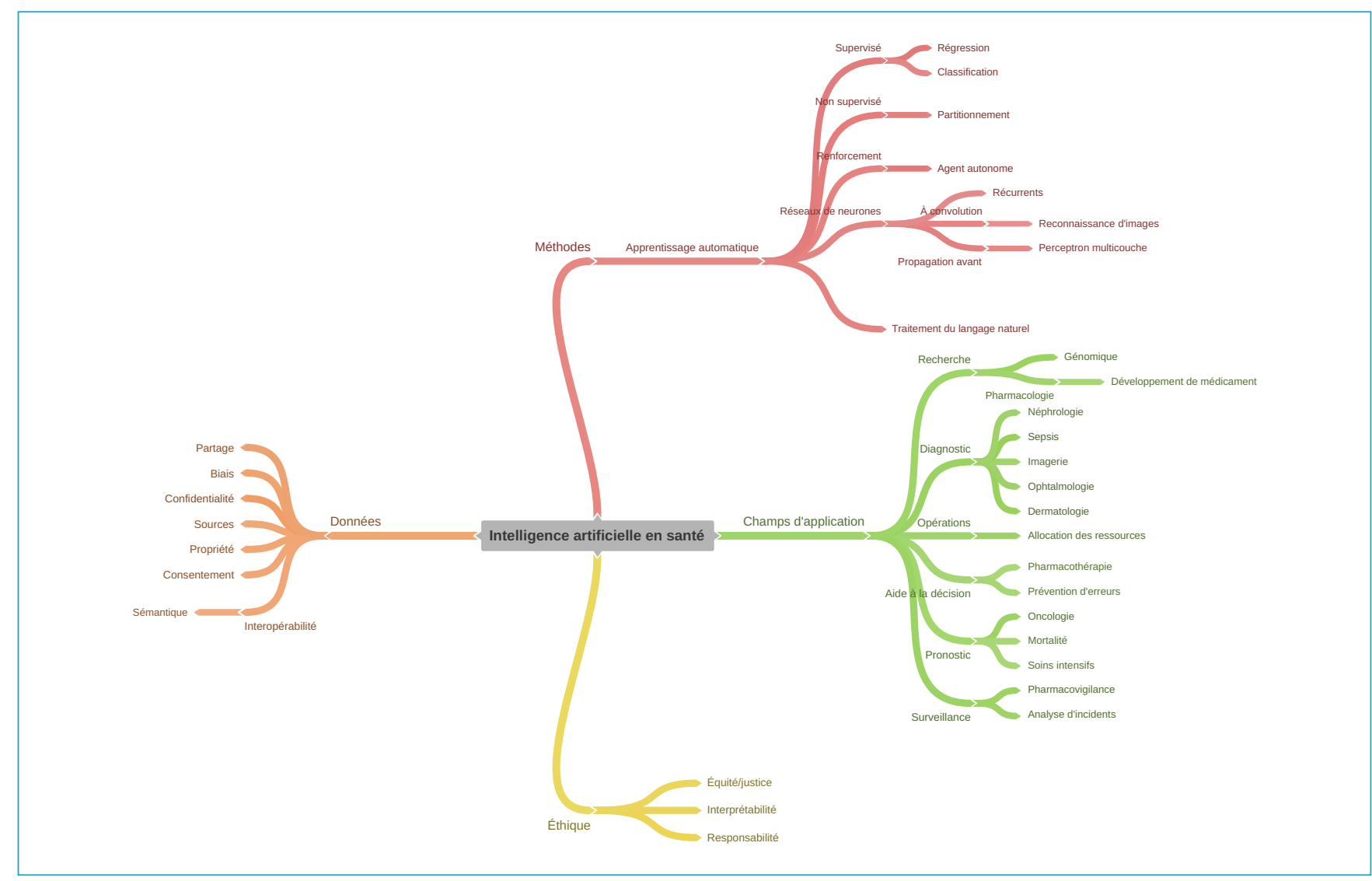

FIGURE 1. Carte heuristique des termes applicables à l'intelligence artificielle en santé.

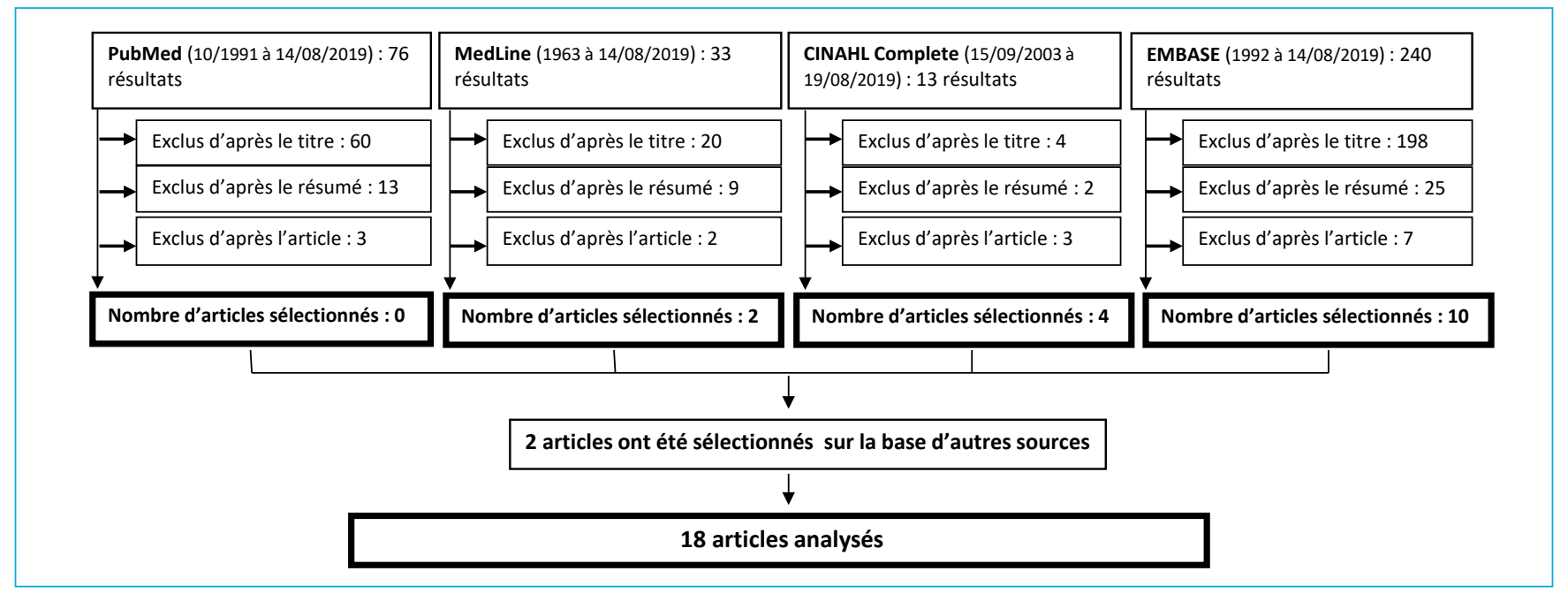

FIGURE 2. Cartographie de sélection des articles sélectionnés et exclus. 
ciblent la prévention dévénements dont le coût est élevé ou qui alourdissent trop le système de santé.

Dans le contexte de la validation d'ordonnances et de la prescription électronique, l'objectif principal des études est d'optimiser l'aide à la décision offerte au moment de l'entrée d'une ordonnance ou de sa validation, pour éviter les alertes inutiles et réduire la désensibilisation aux alertes. On remarque aussi des tentatives de signaler au prescripteur ou au pharmacien les ordonnances statistiquement aberrantes, c'est-à-dire sortant des modèles de prescription habituels. Ceci est un élément nouveau par rapport à laide à la décision classique basée sur des règles préprogrammées.

Les autres études discutent d'analyse de rapports d'incidents, d'analyse d'images pour déterminer le volume de liquide dans des seringues ainsi que de pharmacoéconomie.

Le tableau 1 présente un profil synthèse des études ${ }^{6-23}$ évaluant la faisabilité et l'impact de l'IA en pharmacie.

\section{DISCUSSION}

Cette revue narrative présente une revue originale des études évaluant la faisabilité et l'impact de l'IA en pharmacie. Des 362 articles recensés au préalable, seulement 18 d’entre eux publiés de 2003 à 2019 ont été retenus. Ceci représente une proportion très limitée de ce qui se publie actuellement sur l'IA. À titre d'exemple, l'utilisation du terme $\mathrm{MeSH}$ «Artificial intelligence» dans la base de données PubMed met en évidence 1248 articles publiés en 2000 contre 8091 en 2018.

Le recours à l'IA, peu importe les méthodes utilisées, nécessite un historique de données en quantités suffisantes pour créer des modèles qui pourront être utilisées en temps réel ou de façon asynchrone. En pharmacie, on détient un historique de données important, compte tenu que l'informatisation des ordonnances remonte à plusieurs décennies et que les données recueillies ne sont généralement pas détruites au fil du temps. Normalement, une pharmacie d'officine ou d'hôpital peut traiter de 500 à 1500 ordonnances par jour. Cinq études sur 18 ont été menées sur des données issues de la pratique en pharmacie communautaire.

La revue narrative met en évidence différentes approches méthodologiques de prédiction, par exemple l'utilisation de réseaux de neurones $(n=4)$, des arbres boostés $(n=2)$ ou de régression logistique $(n=2)$. Certaines études comparent ou combinent plus d'une approche. Dans huit études, l’approche utilisée n’est pas décrite. Ceci n'est pas étonnant étant donné que certains auteurs ne veulent pas décrire leur approche en détail, parce quelle pourrait être intégrée dans des produits commerciaux vendus par la suite aux cliniciens et aux établissements de santé. Par contre, certains groupes appellent maintenant à la transparence des techniques utilisées, afin que les cliniciens soient en mesure de comprendre comment sont générées les prédictions de ces outils et qu'ils voient clairement les limites de ces techniques ${ }^{24}$. En outre, les données recueillies ne permettent pas de déterminer les approches à privilégier. Lapproche optimale doit être déterminée au cas par cas selon le problème clinique en question.

La revue narrative met en évidence différents domaines d'utilisation (p. ex. pharmacovigilance, priorisation des patients, adhésion thérapeutique, validation des ordonnances). Le recours à l'IA est émergent et il est encore trop tôt pour déterminer les domaines où les retombées seront plus intéressantes pour la pratique pharmaceutique. De façon générale, on peut dire qu'il est pertinent d'explorer des modèles de prédiction si lon détient un bon ensemble de données et si l'on a déterminé un besoin de prédiction pour soutenir le travail du pharmacien ou de soignants en santé.

Parmi les études recueillies, au moins huit s'intéressent aux maladies chroniques. Une part importante de la population est atteinte de maladies chroniques, qui accaparent une proportion importante des ressources humaines, matérielles et financières dans le domaine de la santé. Les chercheurs font le pari que l'utilisation de l'IA dans le cadre des maladies chroniques pourrait, si les modèles développés ont une bonne capacité prédictive, avoir un jour un impact favorable sur les ressources nécessaires, compte tenu du grand nombre de patients atteints dans le monde. De façon générale, le suivi des maladies chroniques repose sur un nombre circonscrit de paramètres cliniques et de médicaments par affection et il pourrait être intéressant de prédire les médicaments, les tests de laboratoires et les suivis pertinents à effectuer afin de réduire les risques de morbidité, de réhospitalisation et de mortalité. Plusieurs études recensées dans la revue s'intéressent à l'adhésion au traitement dans le contexte des maladies chroniques, afin de prioriser les interventions de pharmaciens pour les patients davantage exposés à un risque de non-adhésion. Compte tenu des conséquences importantes en termes de complications médicales et des coûts de santé liés à la non-adhésion, ce type d'utilisation de l'IA par les pharmaciens a certainement du potentiel ${ }^{25}$.

L'utilisation du terme IA nest pas forcément toujours appropriée. Il est peut-être tentant de mentionner l'expression " intelligence artificielle " dans le titre ou le résumé d'un article afin d'attirer l'attention, même si le sujet ne repose pas sur l'utilisation réelle de l'IA. Notre revue documentaire nous a permis de voir que certains articles présentés sous cette expression portaient plutôt sur des outils d'aide à la décision, l'utilisation d'algorithmes ou de règles permettant de prioriser des patients, sur des conditions cliniques, des médicaments ou d'autres paramètres dans le travail du pharmacien. Plusieurs logiciels intègrent des outils d'aide à la décision (p. ex. afficher une alerte au pharmacien qui valide une ordonnance si la clairance à la créatinine est inférieure à un seuil d’alerte prédéterminé pour ce médicament, afficher une alerte en présence d'une duplication, d'une interaction médicament-médicament ou d'une allergie) sans que ceci soit du domaine de l'IA. À notre avis, l'utilisation de l'IA représente une véritable avancée dans la pratique 


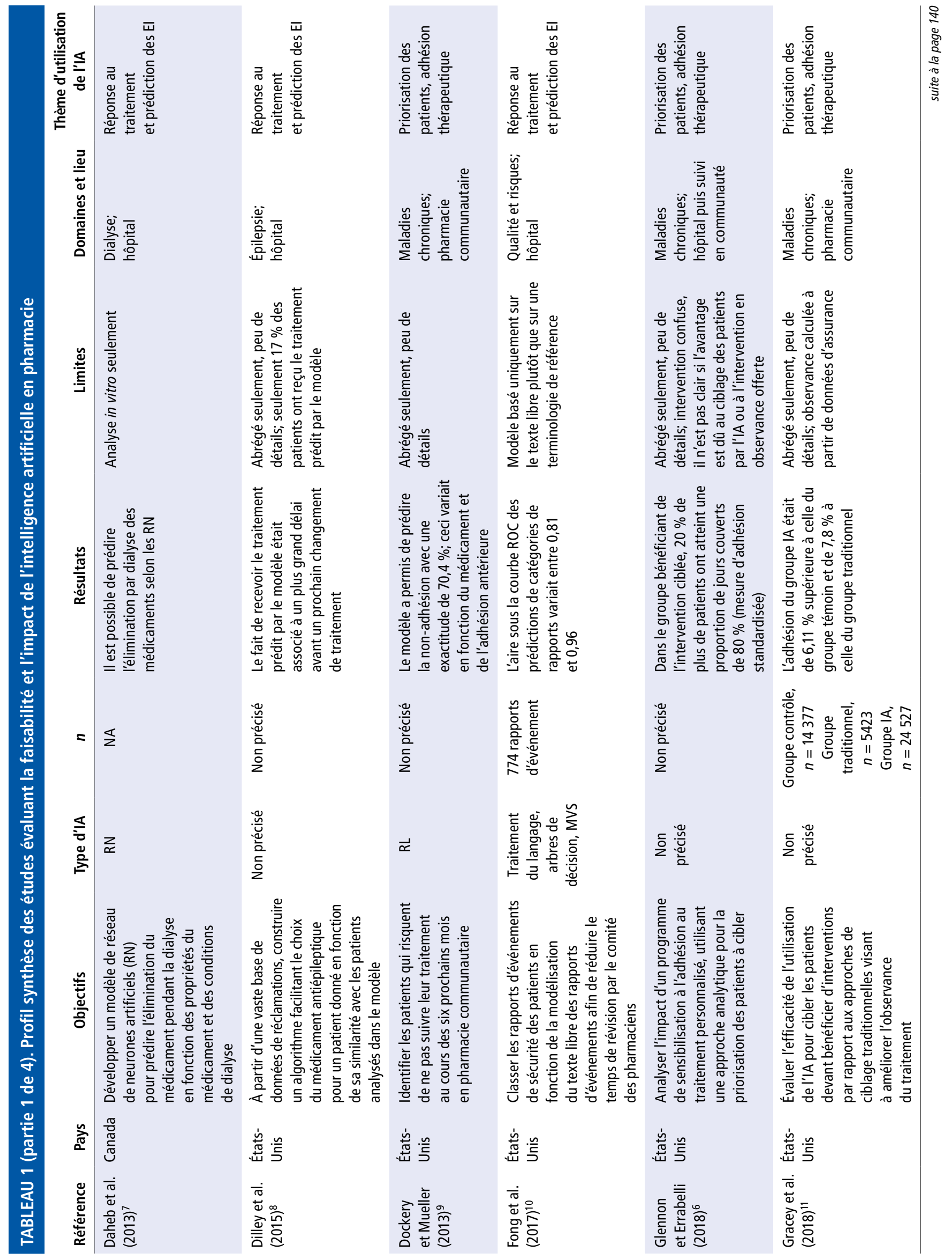




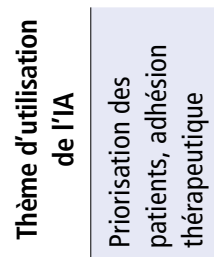

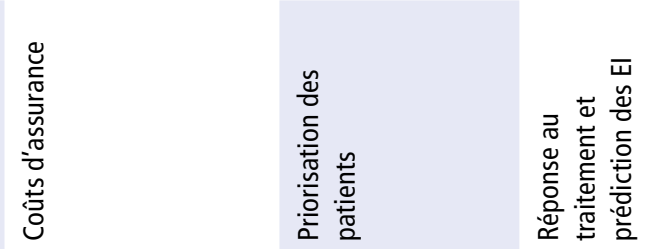

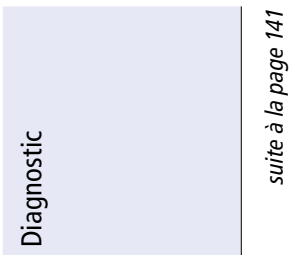

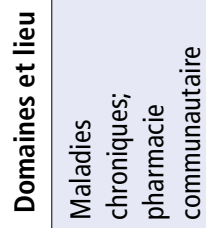

害

๕ัّ

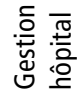

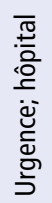

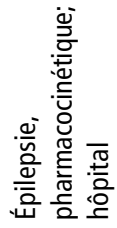

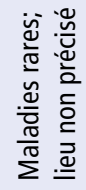

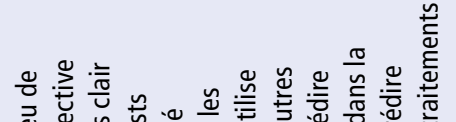

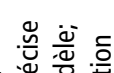

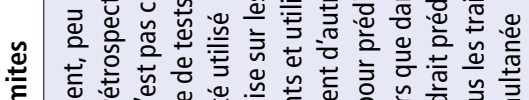

응

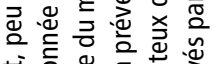

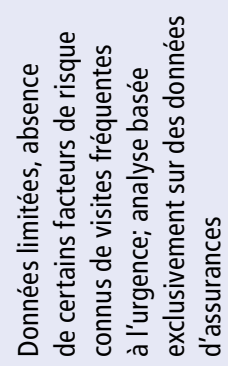

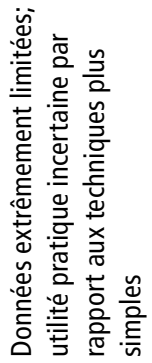

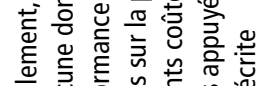

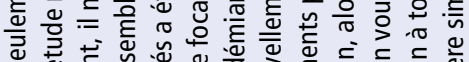

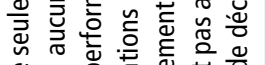

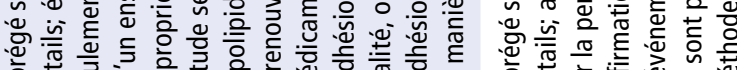

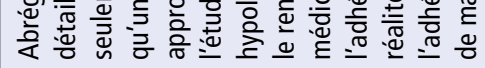
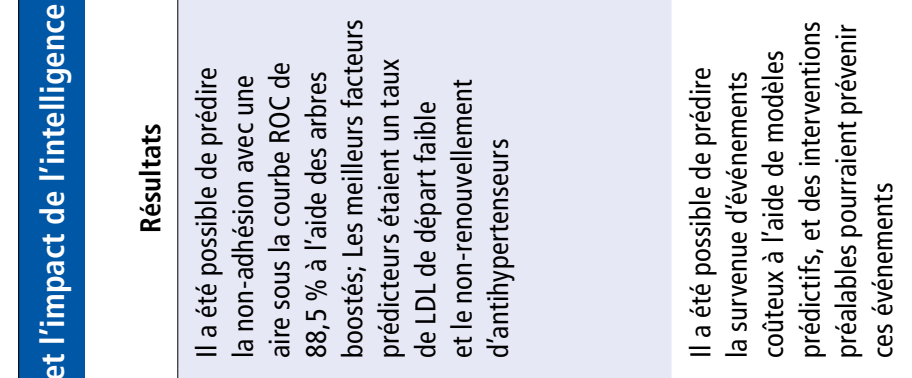

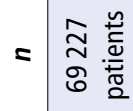

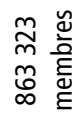

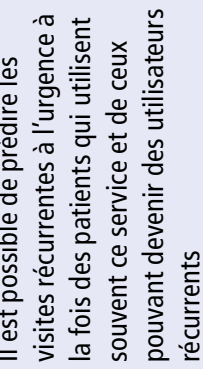

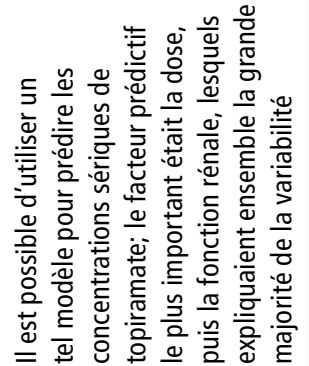

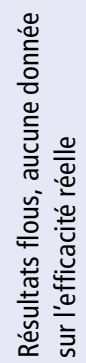

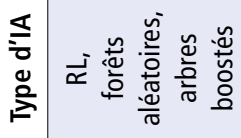

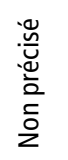

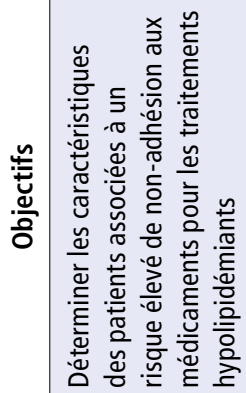

总言

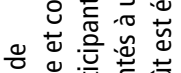

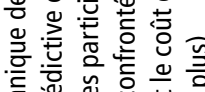

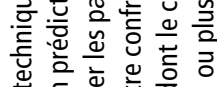

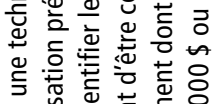

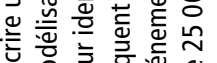

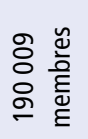

œ

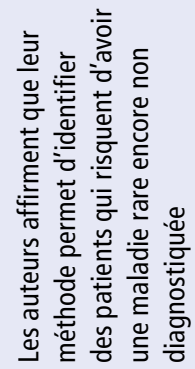

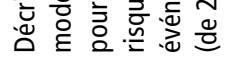

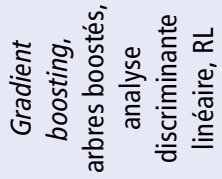

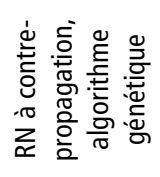

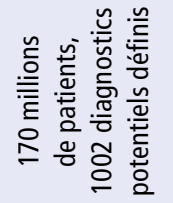

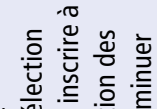

g

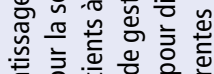

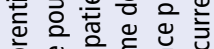

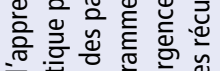

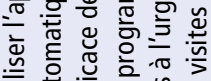

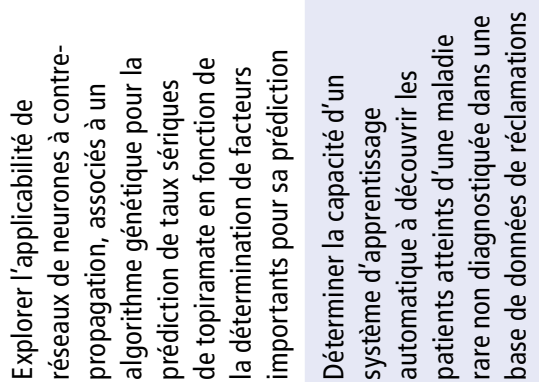

苍 崩

悹

营

产

总

离

商 $\frac{m}{\frac{0}{0}}$

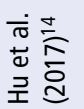

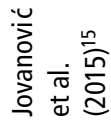

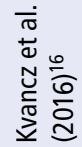




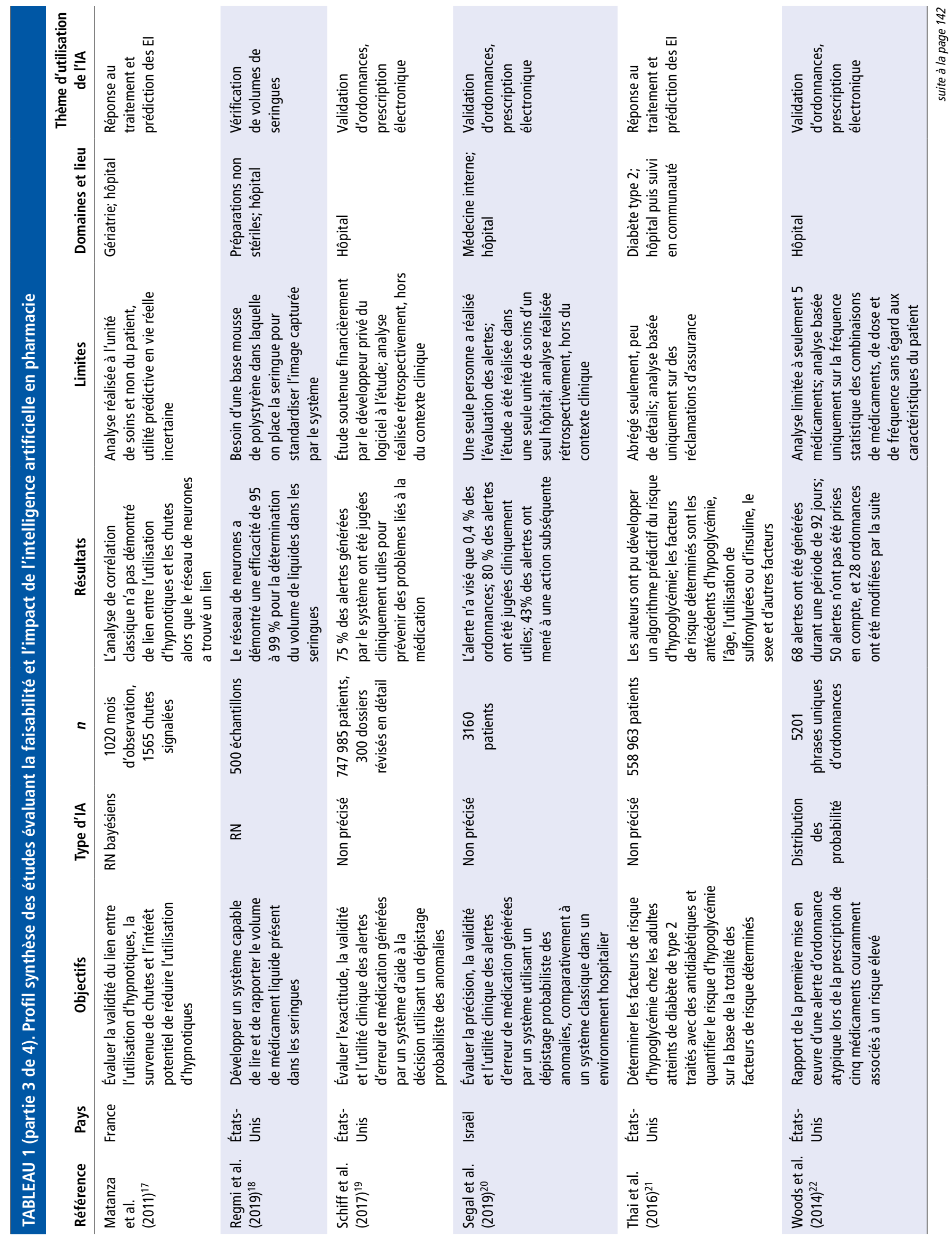




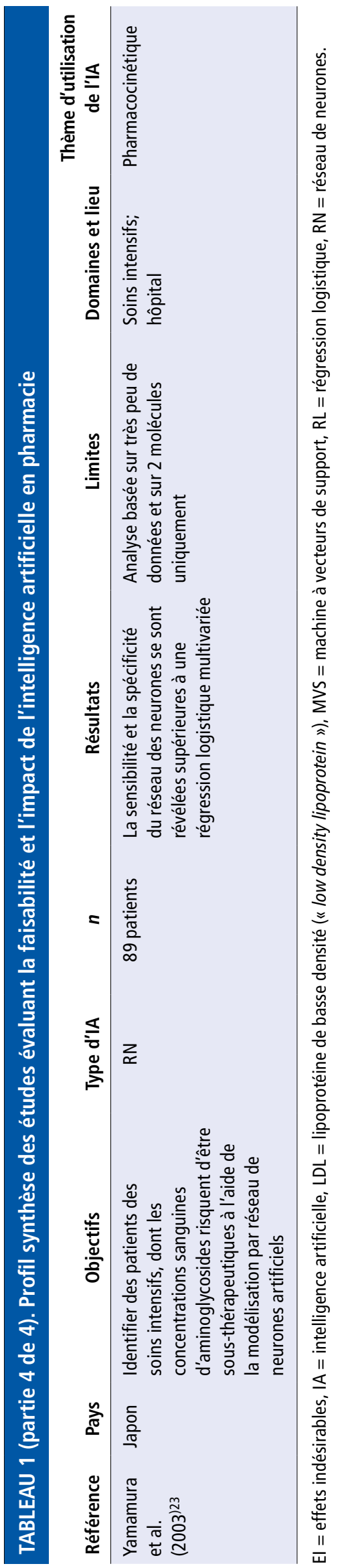

pharmaceutique et il est essentiel que les pharmaciens s'y intéressent. Par exemple, l'American Society of HealthSystem Pharmacists a publié en 2006 (mise à jour en 2016) un énoncé afin que chaque département de pharmacie d'un établissement de santé se dote d'un pharmacien qui se consacre à l'informatique clinique ${ }^{26}$.

Cette revue narrative confirme lémergence de l'IA en pharmacie. Ces articles constituent la pointe de l'iceberg et de nombreuses initiatives émergent un peu partout dans le monde. Il semble nécessaire queles pharmaciens s'y intéressent et que des collaborations sétablissent entre chercheurs en IA et cliniciens. Ce type de collaboration existe au quotidien, mais il génère encore peu d'applications cliniques utilisées en pratique. Les centres de recherche, les facultés de pharmacie, les ministères de la Santé, les établissements de santé, les chaînes et bannières devraient s'intéresser à l'IA. Bien que ce fait soit peu évoqué dans les articles de la présente étude, l'IA ne peut progresser sans le partage de données à des experts en modélisation et prédiction. Le partage de ces données, même anonymisées, n’est pas forcément permis ou sécuritaire compte tenu des enjeux de protection des renseignements personnels. Une réflexion sur les exigences juridiques entourant la confidentialité s'impose pour que les données disponibles en pharmacie soient exploitées de façon éthique, sécuritaire et adéquate.

Cette revue narrative comporte des limites. La consultation n’a porté que sur quatre bases de données; il serait intéressant de consulter d'autres bases de données, ciblant davantage le domaine de l'IA, dont la recherche fondamentale (p. ex. arXiv) et les publications découlant de conférences et de regroupements de chercheurs du domaine (p. ex. NeurIPS, ICML, ICLR, MLHC). Les stratégies de recherche utilisées ciblaient spécifiquement le critère de "pharmacie " ou de "pratique pharmaceutique». Notre recherche a mis en évidence des articles pertinents qui ne comportaient pas le terme «pharmacy» mais dont le contenu aurait pu sappliquer à la pratique pharmaceutique. Par exemple, Tomašev et collab. se sont intéressés à la prédiction de la survenue de l’insuffisance rénale aigüe $\mathrm{e}^{27}$. Les médicaments sont évidemment un facteur de risque majeur de cette complication, de même qu'une réduction importante de la fonction rénale affecte lélimination d'un grand nombre de médicaments. Ce type de modèle pourrait être grandement utile à la pratique pharmaceutique. Chen et collab. se sont intéressés à la prédiction des ordonnances futures en fonction des ordonnances passées du patient ${ }^{28}$. Zhao et collab. se sont intéressés à la détection d'effets indésirables médicamenteux à partir de dossiers électroniques ${ }^{29}$. Il pourrait être utile de faire une sélection de concepts et de termes pertinents à lexercice de la pharmacie afin de recenser davantage d'articles intégrant l'IA en santé, qui sont importants pour la pratique pharmaceutique. Il pourrait être intéressant en outre d'utiliser d'autres termes, tels que : apprentissage automatique et machine learning, pour accroître le nombre d'articles potentiellement pertinents. 


\section{CONCLUSION}

Cette revue narrative met en évidence 18 études évaluant la faisabilité et l'impact de l'IA en pharmacie. Ces études mettent en relief différentes approches méthodologiques et divers domaines d'application, en officine comme en établissement de santé. Il est encore trop tôt pour prédire les retombées de l'IA en pharmacie, mais ces études soulignent l'importance de s'y intéresser.

\section{Références}

1. Intelligence artificielle. Dans : Fiche terminologique. Office québécois de la langue française; 2017 [page consultée le 25 septembre 2019]. [en ligne] : http://gdt.oqlf.gouv.qc.ca/ficheOqlf.aspx?Id_Fiche=8385376

2. Une intelligence artificielle bien réelle : les termes de l'IA. Dans : Lexiques et vocabulaires : Intelligence artificielle. Office québécois de la langue française; 2018 [page consultée le 25 septembre 2019]. [en ligne] : https:// www.oqlf.gouv.qc.ca/ressources/bibliotheque/dictionnaires/vocabulaireintelligence-artificielle.aspx

3. Chartrand G, Cheng PM, Vorontsov E, Drozdzal M, Turcotte S, Pal CJ, et al. Deep learning: a primer for radiologists. Radiographics. 2017;37(7): 2113-31.

4. IBM Watson for oncology: overview. IBM; [page consultée le 25 septembre 2019]. [en ligne] : https://www.ibm.com/us-en/marketplace/ clinical-decision-support-oncology

5. Flynn A. Using artificial intelligence in health-system pharmacy practice: finding new patterns that matter. Am J Health Syst Pharm. 2019; 76(9):622-7.

6. Glennon J, Errabelli D. Improving medication adherence: critical components for effective interventions [abrégé U12]. J Manag Care Spec Pharm. 2018;24(4-a Suppl):S97.

7. Daheb K, Lipman ML, Hildgen P, Roy JJ. Artificial neural network modeling for drug dialyzability prediction. J Pharm Pharm Sci. 2013; 16(5):665-75

8. Dilley C, Rozen-Zvi M, Harrington J, Goldschmidt Y, Clark C, Fritz P, et al. Antiepileptic drug therapy and model predictions of treatment success [abrégé 2.033]. Epilepsy Curr. 2015;15(1 Suppl):197.

9. Dockery JD, Mueller RL. Predicting medication adherence using retail pharmacy data [abrégé]. Value Health. 2013;16(3):A43.

10. Fong A, Harriott N, Walters DM, Foley H, Morrissey R, Ratwani RR. Integrating natural language processing expertise with patient safety event review committees to improve the analysis of medication events. Int J Med Inform. 2017;104:120-5.

11. Gracey B, Jones CA, Cho D, Conner S, Greene E. Improving medication adherence by better targeting interventions using artificial intelligence-a randomized control study [abrégé]. Value Health. 2018;21 Suppl 1:S76.

12. Hill JW, Rane PB, Hinea DM, Patel J, Harrison DJ, Wade RL. Predictive analysis for identifying patient characteristics associated with primary medication nonadherence for lipid lowering therapies [abrégé]. Value Health. 2017;20(9):A402-3.

13. Holloway J, Guh S, Washington V, Cantrell D, Chaisson J, Tisdale K, et al. A predictive modeling approach that accurately identifies members with the likelihood of having a future costly event [abrégé]. Value Health. 2018;21 Suppl:S219-20.

14. $\mathrm{Hu} \mathrm{X}$, Barnes S, Bjarnadottir M, Golden B. Intelligent selection of frequent emergency department patients for case management: a machine learning framework based on claims data. IISE Trans Healthc Syst Engineer. 2017;7(3):130-43. doi: 10.1080/24725579.2017.1351502

15. Jovanović M, Sokić D, Grabnar I, Vovk T, Prostran M, Erić S, et al. Application of counter-propagation artificial neural networks in prediction of topiramate concentration in patients with epilepsy. J Pharm Pharm Sci. 2015;18(5):856-62.

16. Kvancz DA, Sredzinski MN, Tadlock CG. Predictive analytics: a case study in machine-learning and claims databases. Am J Pharm Benefits. 2016;8(6):214-9.
17. Matanza D, Delhome C, Bernard A, Bourguignon L. Falls in the elderly and use of hypnotics: a neural network analysis [abrégé MS-49]. J Clin Pharm. 2011;33:358.

18. Regmi HK, Nesamony J, Pappada SM, Papadimos TJ, Devabhaktuni V. A system for real-time syringe classification and volume measurement using a combination of image processing and artificial neural networks. J Pharm Innov. 2019;14:341-58.

19. Schiff GD, Volk LA, Volodarskaya M, Williams DH, Walsh L, Myers SG, et al. Screening for medication errors using an outlier detection system. J Am Med Inform Assoc. 2017;24(2):281-7.

20. Segal G, Segev A, Brom A, Lifshitz Y, Wasserstrum Y, Zimlichman E Reducing drug prescription errors and adverse drug events by application of a probabilistic, machine-learning based clinical decision support system in an inpatient setting. J Am Med Inform Assoc. 2019;26(12):1560-5.

21. Thai N, Wei L, Anderson J, Alas V, Zhou S, Berhanu P, et al. Novel predictive modeling identifies and quantifies factors that predict the risk of hypoglycemia in patients with type 2 diabetes [abrégé E26]. J Manag Care Spec Pharm. 2016;22(10-a Suppl):S37.

22. Woods AD, Mulherin DP, Flynn AJ, Stevenson JG, Zimmerman CR, Chaffee BW. Clinical decision support for atypical orders: detection and warning of atypical medication orders submitted to a computerized provider order entry system. J Am Med Inform Assoc. 2014;21(3):569-73.

23. Yamamura S, Takehira R, Kawada K, Nishizaea K, Katayama S, Hirano $\mathrm{M}$, et al. Application of artificial neural network modelling to identify severely ill patients whose aminoglycoside concentrations are likely to fall below therapeutic concentrations. J Clin Pharm Ther. 2003;28(5):425-32.

24. Van Calster B, Wynants L, Timmerman D, Steyerberg EW, Collins GS. Predictive analytics in health care: how can we know it works? J Am Med Inform Assoc. 2019;26(12):1651-4.

25. Osterberg L, Blaschke T. Adherence to medication. N Engl J Med. 2005; 353(5):487-97.

26. ASHP statement on the pharmacist's role in clinical informatics. Am J Health Syst Pharm. 2016;73(6):410-3

27. Tomašev N, Glorot X, Rae JW, Zielinski M, Askham H, Saraiva A, et al. A clinically applicable approach to continuous prediction of future acute kidney injury. Nature. 2019;572(7767):116-9.

28. Chen JH, Goldstein MK, Asch SM, Mackey L, Altman RB. Predicting inpatient clinical order patterns with probabilistic topic models vs conventional order sets. J Am Med Inform Assoc. 2017;24(3):472-80.

29. Zhao J, Henriksson A, Asker L, Boström H. Predictive modeling of structured electronic health records for adverse drug event detection. BMC Med Inform Decis Mak. 2015;15 Suppl 4:S1.

Laura Gosselin travaille à I'Unité de recherche en pratique pharmaceutique, Département de pharmacie, CHU Sainte-Justine, Montréal (Québec). Elle est aussi candidate au Pharm. D. à I'Université de Lille, Lille (France).

Maxime Thibault, B. Pharm., M. Sc., travaille à I'Unité de recherche en pratique pharmaceutique, Département de pharmacie, CHU Sainte-Justine Montréal (Québec).

Denis Lebel, M. Sc., FCSHP, travaille à I'Unité de recherche en pratique pharmaceutique, Département de pharmacie, CHU Sainte-Justine, Montréal (Québec).

Jean-François Bussières, B. Pharm., M. Sc., MBA, FCSHP, FOPQ, travaille à I'Unité de recherche en pratique pharmaceutique, Département de pharmacie CHU Sainte-Justine, et à la Faculté de pharmacie, Université de Montréal, Montréal (Québec)

Conflits d'intérêt : Aucune déclaration.

Adresse de correspondance :

Jean-François Bussières

Unité de recherche en pratique pharmaceutique

Département de pharmacie

CHU Sainte-Justine

3175, chemin de la Côte Sainte-Catherine

Montréal QC H3T 1C5

courriel : jean-francois.bussieres.hsj@ssss.gouv.qc.ca

Financement : Aucun reçu. 